\title{
An arterial insufficiency ulcer in an individual with cervical spinal cord injury and hypotension
}

\author{
Michael Stillman ${ }^{1} \cdot$ Steve Williams ${ }^{1}$ \\ Received: 16 April 2020 / Revised: 5 May 2020 / Accepted: 7 May 2020 \\ (C) International Spinal Cord Society 2020
}

\begin{abstract}
Introduction Arterial ulcers develop as a complication of tissue hypoperfusion, ordinarily from atherosclerotic disease, but sometimes due to other medical conditions or trauma. While people with spinal cord injury (SCI) may be predisposed to resting hypotension, and, hence, to inadequate peripheral circulation, there are no reports of arterial ulcers as a secondary effect of SCI.

Case presentation A 54-year-old woman with long standing cervical SCI presented with a nonhealing lateral malleolar ulcer that was complicated by osteomyelitis and unresponsive to biweekly debridement. On examination, she was hypotensive with cool extremities and non-palpable pedal pulses but had no evidence of atherosclerotic disease, cardio-metabolic derangement, or vasculopathy. We treated her with Midodrine with improvement in her peripheral circulation then referred her for surgical intervention.

Discussion To our knowledge, this is the first reported case of an arterial ulcer in a person living with SCI that was most likely caused by resting hypotension. It is possible that individuals with SCI—particularly those with higher-level injuriesare at risk for this condition. Clinicians must be alert to arterial ulcers as a potential complication of SCI with concomitant hypotension.
\end{abstract}

\section{Introduction}

Lower extremity ulcers affect $1 \%$ of the adult population and $3.6 \%$ of individuals over age 65 [1]. Frequently recurrent, these wounds may impair affected individuals' mobility and quality of life and may be associated with a number of medical conditions including vascular disease, neuropathy, obesity, lymphedema, and trauma [1].

Lower extremity arterial ulcers specifically result from reduced blood supply to the legs and feet (2). While this hypoperfusion is ordinarily due to atherosclerotic disease, diabetes mellitus (DM), vasculitis, thalassemia, sickle cell disease, and trauma may all contribute [2,3]. In critically ill individuals, use of vasoconstricting medications (i.e., "pressors") may diminish peripheral circulation and lead to iatrogenic wounds [4]. While clinicians must consider all

Michael Stillman

michael.stillman@jefferson.edu

1 Sidney Kimmel Medical College of Thomas Jefferson University, 1100 Walnut Street, Suite 601, Philadelphia, PA 19107, USA potential etiologies in evaluating arterial ulcers [5], revascularization is considered the "gold standard" method of improving oxygenation of ischemic tissues [5, 6].

People with spinal cord injury (SCI), particularly those with cervical and high thoracic injuries, frequently have resting hypotension [7-9]. This may be due to a number of post-injury physiologic changes including impaired sympathetic function and reduced venous blood return [7], and while the condition may be asymptomatic, it may also result in fatigue, weakness, lightheadedness, dyspnea, and cognitive dysfunction [10-12]. If the hypotension is symptomatic, it is first treated with non-pharmacologic interventions such as compression stockings, abdominal binders, and increased fluid intake. If these measures prove ineffective, however, pharmacologic treatments such as Florinef (a volume expander) and Midodrine (a vasoconstrictor) may be used, either as monotherapy or in combination [13].

Given that people with SCI are frequently hypotensive, it would stand to reason that they are at increased risk for arterial ulcers. However, this association has never been addressed or documented in the literature. In this manuscript, we report the case of a woman with chronic cervical 
SCI and resting hypotension who had a nonhealing arterial ankle ulcer that was successfully managed with a combination of surgical debridement and Midodrine.

\section{Case presentation}

A 54-year-old woman with an American Spinal Injury Association (AIS) A C6 SCI for 36 years was seen in our clinic to establish care. She had been seen by a primary care physician in her home community for a number of years, but wished to transfer care to a center with expertise in caring for individuals with SCI.

The patient felt well and had no significant medical history apart from her SCI. However, she complained of a nonhealing ulcer of her right ankle for nearly one year. She had been seen biweekly by a local surgeon who had been debriding the wound bed, and an MRI of the joint had been "suggestive, but not diagnostic" of osteomyelitis. Despite these interventions, she had noted no improvement in the wound. She was not taking any prescription medications, could not recall a precipitating trauma, denied fever, chills, or weight loss, and had not had any small or large joint swelling or new rashes. Her cardiopulmonary and gastrointestinal reviews of systems were noncontributory.

On examination, the patient had a seated blood pressure of $86 / 60 \mathrm{mmHg}$ and a pulse of 64 beats per minute (BPM). She weighed 136 pounds and had an oxygen saturation of $97 \%$ on room air. She appeared well, had normal pulmonary and abdominal examinations, but had non-palpable dorsalis pedis and posterior tibialis pulses bilaterally with cool pale extremities. She had $1+$ popliteal and femoral pulses and no appreciable bruits of the femoral arteries, the renal arteries, or the abdominal aorta. She had an unstageable ulcer over her right lateral malleolus that was $2 \mathrm{~cm}$ across, penetrating into subcutaneous tissues, and without visible bone. We deemed the ulcer "unstageable" as, despite multiple attempts, we were never able to obtain the patient's wound care notes, and could not say with certainty that it had never involved bone. The wound bed was without pus and the surrounding tissues were mildly swollen but nonerythematous. Her blood counts, basic metabolic panel, lipid profile (low-density lipoprotein of $110 \mathrm{mg} / \mathrm{dL}$ ), and coagulation studies were all normal.

We obtained an ankle brachial index, which was normal (0.92 and 0.91 at the right and left ankles, respectively), and a triple phase bone scan showed "increased flow pool and bone activity" to the right lower malleolus, potentially consistent with osteomyelitis. We initiated Midodrine at a dose of $10 \mathrm{mg}$ twice daily, and at a 3 week follow-up appointment the patient's seated blood pressure was $115 / 70$ $\mathrm{mgHg}$ with a pulse of $68 \mathrm{BPM}$. While her pedal pulses were all $1+$ and her legs were warm to the touch, her ulcer remained similar in size and appearance. We referred the patient to an orthopedic surgeon with expertise in caring for people with SCI, and she underwent a wound debridement with excision of infected bone. An intra-operative wound culture showed no white blood cells and grew "very few" Serratia Marcescens that was susceptible to Ceftriaxone, Ciprofloxacin, and Gentamicin. The patient had no postoperative complications, her sutures were removed 3 weeks post-operatively, and she continues to do well. We have chosen to continue her Midodrine to ensure adequate peripheral circulation.

\section{Discussion}

In this report, we describe a 54-year-old woman with long standing AIS A SCI, systemic hypotension, and a nonhealing arterial ulcer whose pre and peri-operative blood pressure and peripheral circulation were enhanced by using Midodrine. Of note, the patient gave permission to the authors to report on and publish her case.

Based on history, reviews of systems, physical examination, and laboratory and vascular studies, the patient did not have any co-morbidities such as atherosclerosis, diabetes, dyslipidemia, or vasculitis that could have predisposed her to arterial ulcers. In addition, while she did have osteomyelitis, there is no literature suggesting that that condition-in the absence of abscess-inhibits wound closure. In considering this case, then, we concluded that the patient's hypotension was the main cause of her inability to achieve wound closure. Interestingly, while the issue of hypotension and wounds in SCI has not been explicitly evaluated, a recent report noted a relationship between pressure ulcers and hypotension in older hospitalized patients who do not have SCI [14].

While it was not unreasonable for the patient's wound care specialist to remove devitalized tissue from her ulcer bed, it is recommended that debridement of arterial ulcers be delayed until blood flow is improved or re-established [5]. Hence, the conservative care she was receiving would never have led to wound closure and healing without pharmacological blood pressure support.

In reading the major reviews of cardiovascular dysregulation following SCI, we have not found mention of arterial ulcers. It is possible that these wounds are simply not that common among people with SCI, but it is also possible that clinicians are failing to properly diagnose them, or to recognize arterial insufficiency as a major contributing factor. Given the risks of hypotension, accelerated atherogenesis [15, 16], and peripheral vascular disease in SCI [17], we ought to all be aware of this ulcer type. In assessing this patient, we were unable to determine whether her history of hypotension was long standing or a more recent phenomenon. This information, which is 
included in the International Standards for the Assessment of Autonomic Function post SCI [18], would have been useful.

In conclusion, this case indicates that people with SCI and hypotension may be at risk for lower extremity arterial ulcers, and that they may not adequately heal-even in the setting of proper wound care-without pharmacologic blood pressure support.

\section{Data availability}

All data generated or analyzed during this report are included in this published article.

\section{Compliance with ethical standards}

Conflict of interest The authors declare that they have no conflict of interest.

Publisher's note Springer Nature remains neutral with regard to jurisdictional claims in published maps and institutional affiliations.

\section{References}

1. London NJ, Donnelly R. ABC of arterial and venous disease. Ulcerated Low limb BMJ. 2000;320:1589-91.

2. Grey JE, Harding KG, Enoch S. Venous and arterial leg ulcers. BMJ. 2006;332:347-50.

3. Barshes NR, Belkin M.MOVIE Study Collaborators A framework for the evaluation of "value" and cost-effectiveness in the management of critical limb ischemia. J Am Coll Surg. 2011;213:552-66.e5.

4. Cox J. Pressure ulcer development and vasopressor agents in adult critical care patients: a literature review. Ostomy Wound Manag. 2013;59:50-4-56-60.

5. Hopf HW, Ueno C, Aslam R, Burnand K, Fife C, Grant L, et al. Guidelines for the treatment of arterial insufficiency ulcers. Wound Repair Regen. 2006;14:693-710.
6. Stranden E, Slagsvold CE. Arterial ischemic ulcers. Tidsskr Nor Laegeforen. 2005;125:895-8.

7. Popa C, Popa F, Grigorean VT, Onose G, Sandu AM, Popescu M, et al. Vascular dysfunctions following spinal cord injury. J Med Life. 2010;3:275-85.

8. Partida E, Mironets E, Hou S, Tom VJ. Cardiovascular dysfunction following spinal cord injury. Neural Regen Res. 2016;11:189-94.

9. Biering-Sorensen F, Biering-Sorensen T, Liu N, Malmqvist L, Wecht JM, Krassioukov A. Alterations in cardiac autonomic control in spinal cord injury. Auton Neurosci. 2018;209:4-18.

10. Sclater A, Alagiakrishnan K. Orthostatic hypotension. A primary care primer for assessment and treatment. Geriatrics. 2004;59:22-27.

11. Frisbie JH, Steele DJ. Postural hypotension and abnormalities of salt and water metabolism in myelopathy patients. Spinal Cord. 1997;35:303-7.

12. Sachdeva R, Gao F, Chan CCH, Krassioukov AV. Cognitive function after spinal cord injury: a systematic review. Neurology 2018;91:611-21.

13. Krassioukov A, Eng JJ, Warburton DE, Teasell R.Spinal Cord Injury Rehabilitation Evidence Research Team A systematic review of the management of orthostatic hypotension after spinal cord injury. Arch Phys Med Rehabil. 2009;90:876-85.

14. Man SP, Au-Yeung TW. Hypotension is a risk factor for new pressure ulcer occurrence in older patients after admission to an acute hospital. J Am Med Dir Assoc. 2013;14:627. e1-627.e5

15. Lee CS, Lu YH, Lee ST, Lin CC, Ding HJ. Evaluating the prevalence of silent coronary artery disease in asymptomatic patients with spinal cord injury. Int Heart J. 2006;47:325-30.

16. Orakzai SH, Orakzai RH, Ahmadi N, Agrawal N, Bauman WA, Yee $\mathrm{F}$, et al. Measurement of coronary artery calcification by electron beam computerized tomography in persons with chronic spinal cord injury: evidence for increased atherosclerotic burden. Spinal Cord. 2007;45:775-9.

17. Bell JW, Chen D, Bahls M, Newcomer SC. Evidence for greater burden of peripheral arterial disease in lower extremity arteries of spinal cord-injured individuals. Am J Physiol Heart Circ Physiol. 2011;301:H766-72.

18. Alexander MS, Biering-Sorensen F, Bodner D, Brackett NL, Cardenas D, Charlifue S, et al. International standards to document remaining autonomic function after spinal cord injury. Spinal Cord. 2009;47:36-43. 